\title{
Phase control of magnetic state of graphite thin films by electric field
}

\author{
Minoru Otani, ${ }^{1,4}$ Yoshiteru Takagi, ${ }^{2,4}$ Mikito Koshino, ${ }^{3}$ and Susumu Okada ${ }^{2,4, a)}$ \\ ${ }^{1}$ Research Institute for Computational Sciences, National Institute of Advanced Industrial Science \\ and Technology (AIST), Tsukuba 305-8568, Japan \\ ${ }^{2}$ Graduate School of Pure and Applied Sciences and Center for Computational Sciences, \\ University of Tsukuba, 1-1-1 Tennodai, Tsukuba 305-8571, Japan \\ ${ }^{3}$ Department of Physics, Tokyo Institute of Technology, 2-12-1 Oh-okayama, Meguro-ku, \\ Tokyo 152-8551, Japan \\ ${ }^{4}$ Japan Science and Technology Agency, CREST, 5 Sanbancho, Chiyoda-ku, Tokyo 102-0075, Japan
}

(Received 21 February 2010; accepted 27 May 2010; published online 15 June 2010)

\begin{abstract}
Based on first-principle total-energy calculations, we have found that by applying an external electric field it is possible to control the magnetic state of graphite thin film with the rhombohedral stacking arrangement. When exposed to a moderate electric field normal to the film, the surface of a thin film of rhombohedral graphite undergoes a magnetic phase transition from the antiferromagnetic state to the ferromagnetic state. The polarized electron spin is primarily distributed in the bottommost layer of the film, which forms the interface with the negative electrode. The amount of polarized electron spin is calculated to be $0.067 \mu_{\mathrm{B}} / \mathrm{nm}^{2}$. The ferromagnetic ordering with the characteristic distribution of the polarized electron spin opens the possibility of using graphite thin films in electronic devices with spin degree of freedom. (C) 2010 American Institute of Physics. [doi:10.1063/1.3455069]
\end{abstract}

Following the synthesis of single-layer graphite (graphene), ${ }^{1}$ many theoretical and experimental efforts were devoted to this legendary carbon allotrope, which possesses a two-dimensional (2D) hexagonal network of C atoms. ${ }^{2,3}$ These researches have uncovered the peculiar properties of graphite and graphene that were predicted by previous theoretical works and have also revealed some unusual properties that were unexpected. Graphite is the metal with layered structure, in which the conducting properties are characterized by massless electrons at the Fermi level leading to a high electron mobility through the layer. Because of its properties, graphite and graphene now occupy a premier position not only in low-dimensional sciences but also in nanoscale technologies in the next generation. For example, graphite can work as the conducting channel of field-effect transistors (FETs).

Another source of interest for graphite and graphene is magnetism induced by vacancies, edges, or topological defects. ${ }^{4-7}$ Graphene containing such imperfections exhibits peculiar electronic states around the Fermi level. ${ }^{8-10}$ In conventional solids with covalent character (e.g., $\mathrm{Si}$ and diamond), imperfections basically result in electron states near the Fermi level that are localized around the imperfection because of the unpaired electrons in the unsaturated covalent bonds. For graphene, however, imperfections cause the emergence of another class of electronic states. When graphene flakes or ribbons have zigzag edges, peculiar electronic states emerge at the Fermi energy. They are localized near to but extended along the edges. In part of the Brillouin zone, these states lack dispersion along the edge directions. ${ }^{8-10}$ Halffilled flatband states lead to certain magnetic ordering on the graphene flakes; polarized electron spins are ferromagnetically aligned along each edge and antiferromagnetically coupled between edges. ${ }^{7}$ This state is now known as the edge state. $^{8-12}$ The magnetism associated with the edge state is not

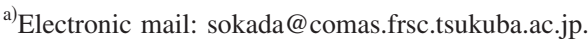

peculiar to graphene flakes with zigzag edges but occurs on the (0001) surfaces of bulk rhombohedral [ABC stacking: Fig. 1(a)] graphite. ${ }^{16}$ In that case, the flatband state emerges in the vicinity of the $K$ point of the two-dimensional hexagonal Brillouin zone, ${ }^{13-17}$ and the surface exhibits ferrimagnetic spin ordering with a magnetic moment of $0.036 \mu_{\mathrm{B}} / \mathrm{nm}^{2} .^{16}$ In the case, although the polarized spins are ferrimagnetically aligned on each surface, the polarized spin on the topmost and the bottommost layers are antiferromagnetically coupled to each other so that the net spin in the slab is zero.

In the present work, based on first-principle total-energy calculations, we demonstrate the possibility of controlling the magnetic phase of thin films of rhombohedral graphite by applying an external electric field normal to graphene layers. Our calculations clearly indicate that the thin films of rhom- (a)

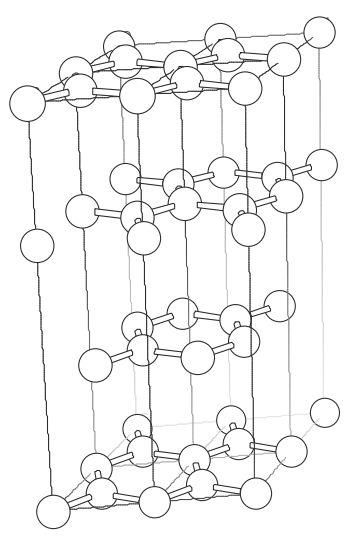

(b)

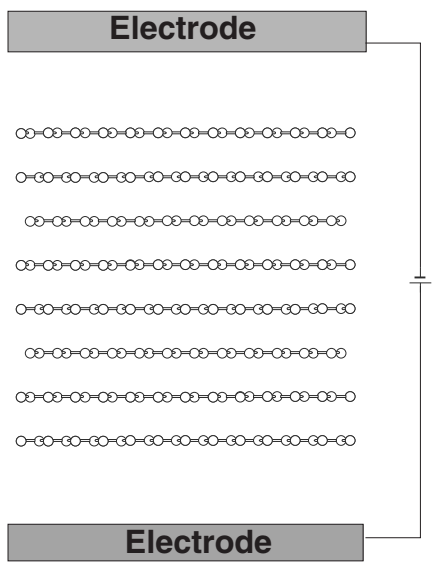

FIG. 1. (a) Atomic structure of graphite with rhombohedral stacking arrangement. (b) A side view of a thin film of rhombohedral graphite comprising eight graphene layers. The topmost and the bottommost layers face the electrodes. 
(a)

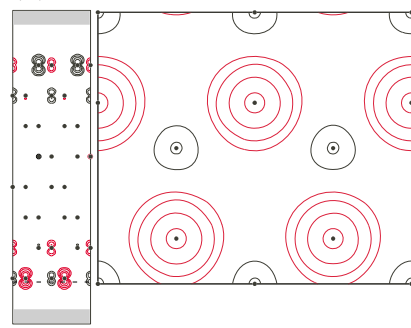

(b)

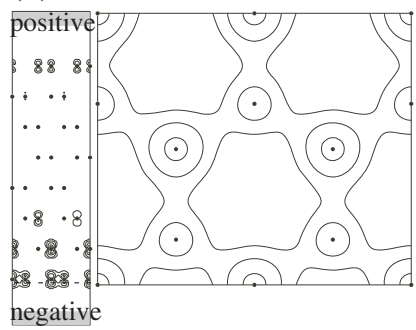

FIG. 2. (Color online) Contour plots of spin density. $\Delta \rho=\rho_{\uparrow}(\boldsymbol{r})-\rho_{\downarrow}(\boldsymbol{r})$ for rhombohedral graphite (a) without and (b) with an external electric field. The solid circles denote the position of $\mathrm{C}$ atoms. Positive and negative values of the spin density are shown by black and gray (red) lines, respectively. Each contour represents twice (or half) the density of the adjacent contour lines. Vertical dotted lines in the left panel of each figure denote the position where the contour plot parallel to the slab is drawn.

bohedral graphite undergo a magnetic phase transition when subjected to a moderate electric field; the electric field transforms the antiferromagnetic state into a ferromagnetic state. The present results open the possibility of using graphite thin films as constituent materials in spin-dependent electronic devices possessing the metal-oxide semiconductor FET structure.

In this work, we present a first-principles total-energy calculation based on the density functional theory. ${ }^{18,19}$ To express the quantum many-body effect on interacting electrons, we use the local spin density approximation (LSDA) with the Perdew-Zunger ${ }^{20}$ functional form. ${ }^{21}$ We adopt the Vanderbilt ultrasoft pseudopotential to describe the electronion interaction. ${ }^{22}$ The valence wave functions and charge density are expanded in a plane-waves basis set with a cutoff energy of 25 Ry and 324 Ry, respectively. In this case, because the flatband region affecting the magnetism on the graphite surface occupies a narrow region in the Brillouin zone, integration over the Brillouin zone is performed using fine equidistant $k$-point meshes, in which $52 \times 52 k$-points are taken in the 2D hexagonal Brillouin zone. The external electric field applied normal to the slab is simulated by the effective screening medium (ESM) method in which the Poisson equation is solved by imposing the open boundary condition normal to the graphite slab. ${ }^{23,24}$ To simulate a thin film of rhombohedral graphite, we use a graphite slab consisting of eight graphene layers with a rhombohedral stacking arrangement. An early experiment has reported that $14 \%$ of natural graphite is rhombohedral graphite. ${ }^{25}$ Thus, the structural model considered here is not fictitious but physically relevant. The slab is then sandwiched between two electrodes with $10.0 \AA$ vacuum regions that are simulated by the ESM [Fig. 1(b)]. For the lateral direction, we impose the periodic boundary condition on $1 \times 1$ unit containing two $\mathrm{C}$ atoms in each layer to simulate infinite graphene layer.

Figure 2(a) shows the spin density of a graphite slab with a rhombohedral stacking arrangement and with no external electric field. In this case, the polarized electron spins are ferrimagnetically aligned on each surface, whereas the polarized spin on the topmost and the bottommost layers are antiferromagnetically coupled to each other. Because of this magnetic ordering, the net magnetic moment in the slab is zero, although each surface possesses a magnetic moment of $0.036 \mu_{\mathrm{B}} / \mathrm{nm}^{2}$.

The magnetic ordering just described is drastically modulated by applying an electric field normal to the slab. Subjected to an electric field of $0.26 \mathrm{~V} / \mathrm{A}$, the rhombohedral graphite slab exhibits ferromagnetic states instead of antiferromagnetic states, as shown in Fig. 2(b). The spin density clearly corroborates the ferromagnetic ordering of the rhombohedral graphite slab. Furthermore, the polarized electron spin is mainly distributed in the bottommost layer, which forms the interface with the negative electrode. The amount of polarized electron spin is calculated to be $0.067 \mu_{\mathrm{B}} / \mathrm{nm}^{2}$. Although the magnetic moment per unit area is less than that of conventional magnetic elements, because the domain extends sufficiently, the net moment is large enough to allow the rhombohedral graphite ferromagnet to be used in the various electronic devices with spin degree of freedom. For instance, the spin-dependent conducting channel is expected to be situated at the surfaces of rhombohedral graphite. In this case, the remarkable stability of the $s p^{2}$ network as well as the 2D distribution of the polarized electron spin would allow robust carrier transportation throughout the slab.

It is important to clarify the origin of ferromagnetic spin ordering on thin films of rhombohedral graphite. Figure 3 shows the electronic energy band and the distribution of the (a)

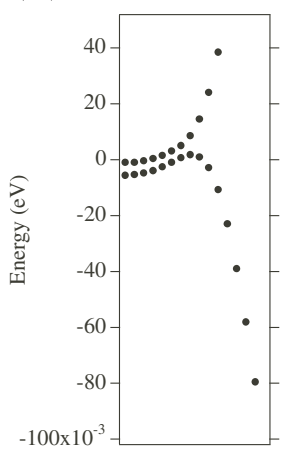

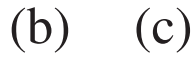

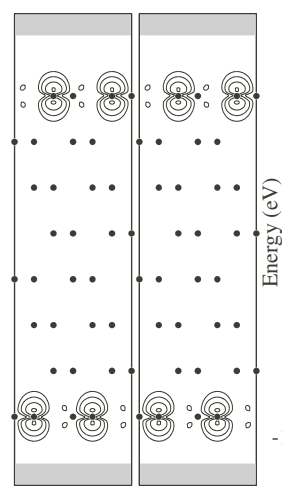

(d)

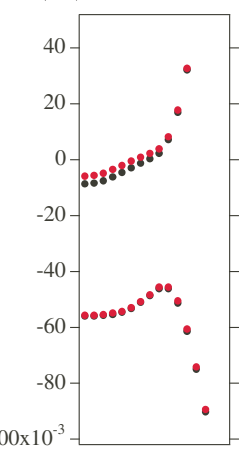

(e)

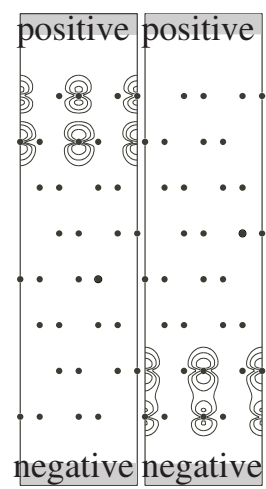

FIG. 3. (Color online) (a) The electronic energy band around the Fermi level in the vicinity of the $K$ point of rhombohedral graphite. Contour plots of the squared wave function of (b) the lower and (c) the upper branches of the flatband state at the $K$ point. (d) The electronic energy band around the Fermi level in the vicinity of the $K$ point of rhombohedral graphite under the influence of an electric field. Contour plots of the squared wave function of (e) the lower and (f) the upper branches of the flatband state at the $K$ point. Energies are measured from the Fermi level energy. Gray (red)- and black-dotted lines denote the electronic energy band for minority and majority spins, respectively. Each contour line represents twice (or half) the density of the adjacent contour lines. 
squared wave function at the $K$ point for a rhombohedral graphite slab with and without an electric field. In the zerofield case, the flatband state emerges near the $K$ point and is split by $5 \mathrm{meV}$ into two branches, one above and one below the Fermi level, for both majority and minority spin states. The wave functions of these states at the $K$ point are completely localized on the outermost layer of the rhombohedral graphite slab. This distribution leads to the antiparallel coupling between the polarized electron spin on the topmost and the bottommost layers.

The situation is drastically altered upon applying an external electric field. We find that the potential deference between the topmost and bottommost layers increases the splitting of the flatband state. The energy gap between the upper and lower branches of the flatband state is $50 \mathrm{meV}$. The upper branch of the flatband state is partially filled by electrons, whereas the lower branch is fully occupied. Because of the partially filled character of the upper branch, it is split by $5 \mathrm{meV}$ into majority- and minority-spin states, which leads to the ferromagnetic spin polarization in the rhombohedral graphite slab. Indeed, the distribution of the wave function corroborates the fact that the state exhibiting exchange splitting is distributed in the bottommost and in the second sublayers facing the negatively charged electrode. The wave function distribution is qualitatively the same as that of the electron spin density.

Finally, we investigate how the magnetic ordering of a rhombohedral graphite slab depends on the external electric field. The ferromagnetic state emerges at the threshold voltage of about $0.2 \mathrm{~V} / \AA$. Above this threshold, the ferromagnetic spin state is the ground state, and the antiferromagnetic spin state is physically irrelevant. At this threshold, the level (electronic energy band) crossing around the Fermi energy occurs between the edge state associated with the antiferromagnetic ordering and the other electron states possessing slightly extended nature [Figs. 3(e) and 3(f)]. Thus, for an external electric field of about $0.2 \mathrm{~V} / \AA$, rhombohedral graphite slabs undergo a magnetic phase transition from the antiferromagnetic state to the ferromagnetic state. On the other hand, it is easy to speculate that the ferromagnetic state disappears under a large external electric field. The large electric field enhances the splitting of the electronic energy band belonging to each graphite layer, which results in the metallic character of the slab.

In summary, we studied the magnetic properties of thin films of rhombohedral graphite exposed to external electric fields. Our first-principle calculations show that the electric field can control the magnetic properties of thin films of rhombohedral graphite. The graphite film undergoes a magnetic phase transition from antiferromagnetic to ferromagnetic when exposed to an electric field normal to the slab. Under the influence of an electric field, the polarized electron spin is primarily distributed in the bottommost layer, which forms the interface with the negative electrode. The amount of the polarized electron spin is calculated to be $0.067 \mu_{\mathrm{B}} / \mathrm{nm}^{2}$. The characteristic distribution of the polarized electron spin in the ferromagnetic state opens the possibility for using graphite thin films as constituent units of electronic devices with spin degree of freedom. For example, the spin-dependent conducting channel is expected to occur at the surface of rhombohedral graphite.

This work was partly supported by CREST, Japan Science and Technology Agency, and a Grant-in-Aid for scientific research from the Ministry of Education, Culture, Sports, Science and Technology of Japan (Grant No. 19054002). Computations were performed on an NEC SX8/4B at the University of Tsukuba, on an NEC SX-8 at the Yukawa Institute of Theoretical Physics, Kyoto University, and on an NEC SX-9 at the Information Synergy Center, Tohoku University.

${ }^{1}$ K. S. Novoselov, A. K. Geim, S. V. Morozov, D. Jiang, Y. Zhang, S. V. Dubonos, I. V. Grigorieva, and A. A. Firsov, Science 306, 666 (2004).

${ }^{2}$ M. S. Dresselhaus and G. Dresselhaus, Adv. Phys. 30, 139 (1981).

${ }^{3}$ A. H. Castro Neto, F. Guinea, N. M. R. Peres, K. S. Novoselov, and A. K. Geim, Rev. Mod. Phys. 81, 109 (2009).

${ }^{4}$ P. O. Lehtinen, A. S. Foster, Y. Ma, A. V. Krasheninnikov, and R. M. Nieminen, Phys. Rev. Lett. 93, 187202 (2004).

${ }^{5}$ Y. Ma, P. O. Lehtinen, A. S. Foster, and R. M. Nieminen, New J. Phys. 6 , 68 (2004).

${ }^{6}$ S. Okada, K. Nakada, K. Kuwabara, K. Daigoku, and T. Kawai, Phys. Rev. B 74, 121412(R) (2006).

${ }^{7}$ S. Okada and A. Oshiyama, Phys. Rev. Lett. 87, 146803 (2001).

${ }^{8}$ M. Fujita, K. Wakabayashi, K. Nakada, and K. Kusakabe, J. Phys. Soc. Jpn. 65, 1920 (1996).

${ }^{9}$ K. Nakada, M. Fujita, G. Dresselhaus, and M. S. Dresselhaus, Phys. Rev. B 54, 17954 (1996).

${ }^{10}$ Y. Miyamoto, K. Nakada, and M. Fujita, Phys. Rev. B 59, 9858 (1999).

${ }^{11}$ Y. Takagi and S. Okada, Surf. Sci. 602, 2876 (2008).

${ }^{12}$ Y. Takagi, J. Phys. Soc. Jpn. 78, 064701 (2009).

${ }^{13}$ F. Guinea, A. H. Castro Neto, and N. M. R. Peres, Phys. Rev. B 73, 245426 (2006).

${ }^{14}$ J. L. Mañes, F. Guinea, and M. A. Vozmediano, Phys. Rev. B 75, 155424 (2007).

${ }^{15}$ M. Koshino and E. McCann, Phys. Rev. B 80, 165409 (2009).

${ }^{16}$ M. Otani, M. Koshino, Y. Takagi, and S. Okada, Phys. Rev. B 81, 161403(R) (2010)

${ }^{17}$ M. Koshino, Phys. Rev. B 81, 125304 (2010).

${ }^{18}$ P. Hohenberg and W. Kohn, Phys. Rev. 136, B864 (1964).

${ }^{19}$ W. Kohn and L. J. Sham, Phys. Rev. 140, A1133 (1965).

${ }^{20}$ J. P. Perdew and A. Zunger, Phys. Rev. B 23, 5048 (1981).

${ }^{21}$ D. M. Ceperley and B. J. Alder, Phys. Rev. Lett. 45, 566 (1980).

${ }^{22}$ D. Vanderbilt, Phys. Rev. B 41, 7892 (1990).

${ }^{23}$ I. Hamada, M. Otani, O. Sugino, and Y. Morikawa, Phys. Rev. B 80, 165411 (2009).

${ }^{24}$ M. Otani and O. Sugino, Phys. Rev. B 73, 115407 (2006).

${ }^{25}$ H. Lipson and A. R. Stokes, Proc. R. Soc. London, Ser. A 181, 101 (1942) 